\title{
Synthesis and properties of some pyrazole derivatives of 1,2,4-triazole-3-thiol
}

\author{
A. S. Hotsulia (D)*A-D,F, S. M. Kulish ${ }^{E}$ \\ Zaporizhzhia State Medical University, Ukraine
}

A - концепція та дизайн дослідження; B - збір даних; C - аналіз та інтерпретація даних; D - написання статті; E - редагування статті;

$\mathrm{F}$ - остаточне затвердження статті

Nitrogen-containing heterocyclic compounds play an important role in the modern pharmaceutical industry. This is due to their significant biological potential. 1,2,4-Triazole and pyrazole are known pharmacophores that are responsible for the formation of a wide range of activities. The construction of the target molecule using 1,2,4-triazole and pyrazole is also interesting in terms of the availability of starting reagents and the simplicity of chemical transformations. The combination of these heterocycles in one molecule allows increasing its opportunities of participation in various biological processes.

The aim of the research was to examine the conditions for obtaining S-alkylderivatives of 5-(5-methylpyrazole)-4-ethyl-1,2,4-triazole-3thiol and to investigate these compounds' properties.

Materials and methods. The first phase of the research involved the use of diethyloxalate, acetone and sodium methylate as starting substances for the synthesis of the intermediate. The obtained methyl 2,4-dioxopentanoate in the following step was converted into 5-methylpyrazole-3-carbohydrazide with the double amount of hydrazine hydrate. The subsequent modification of the molecule implied a stepwise use of the reactions of nucleophilic addition of ethyl isothiocyanate and alkaline cyclization. Thus, the obtained 5-(5-methylpyrazole)-4-ethyl-1,2,4-triazole-3-thiol became subject to an alkylation process. To establish the composition and identify the structure of the isolated substances, ${ }^{1} \mathrm{H}$ NMR and infrared spectra were recorded, as well as qualitative and quantitative indicators of the elemental composition of the synthesized structures were obtained. The individual nature of the presence of substances and the degree of their purity were determined using high performance liquid chromatography with two types of detection: diode-matrix and mass spectrometric.

Results. The synthesis of alkylderivatives of 5-(5-methylpyrazole)-4-ethyl-1,2,4-triazole-3-thiol was carried out and the optimal conditions for the process of obtaining these substances were determined. The structure of the products of chemical transformation is confirmed and the results of the study of its physical properties are recorded.

The results of docking studies allowed to confirm the prospects of the chosen direction of synthetic transformations, which ultimately allowed to determine the biological potential of the obtained compounds. The model enzymes were kinase of the anaplastic lymphoma (code 2XP2), 14-alpha demethylase of the lanosterol (code 3LD6) and cyclooxygenase-1 (code 3N8Y), information on which was obtained from the Protein Structures Database (PDB).

Conclusions. Molecular docking resulted in obtaining data that form a concept of a certain level of probability of synthesized compounds' influence on the activity of these enzyme structures.

Key words: 5-methylpyrazole, 1,2,4-triazole, physical-chemical properties, molecular docking.

Current issues in pharmacy and medicine: science and practice 2020; 13 (3), 318-323

\section{Синтез і властивості деяких піразолпохідних 1,2,4-тріазол-3-тіолу}

А. С. Гоцуля, С. М. Куліш

Нітрогеновмісні гетероциклічні сполуки відіграють важливу роль у сучасній фрармацевтичній галузі. Це пов'язано зі значущим біологічним потенціалом цих сполук. 1,2,4-Тріазол і піразол - відомі фрармакофори, що відповідають за фрормування широкого спектра активностей. Конструювання цільової молекули з використанням 1,2,4-тріазолу та піразолу $є$ також цікавим із погляду доступності вихідних реагентів і простоти хімічних перетворень. Поєднання цих гетероциклів в одній молекулі дає змогу збільшити можливість участі в різноманітних біологічних процесах.

Мета роботи - дослідження умов одержання S-алкілпохідних 5-(5-метилпіразол)-4-етил-1,2,4-тріазол-3-тіолу та вивчення властивостей цих сполук.

ARTICLE

INFO

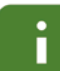

http:l/pharmed.

zsmu.edu.ualarticle/ view/216164
UDC 547.792 '367'77.03/04.057

DOI: $10.14739 / 2409-2932.2020 .3 .216164$

Current issues in pharmacy and medicine: science and practice 2020; 13 (3), 318-323

Key words: 5-methylpyrazole, 1,2,4-triazole, physical-chemical properties, molecular docking.

*E-mail: andrey.goculya@gmail.com

Received: 04.09.2020 // Revised: 14.09.2020 // Accepted: 16.09.2020 
Матеріали та методи. Початковий етап роботи передбачав використання діетилоксалату, ацетону та натрій метилату як вихідних речовин для синтезу проміжного продукту хімічного перетворення. Одержаний метил 2,4-діоксопентаноат на наступному етапі застосований у процесі перетворення у 5-метилпіразол-3-карбогідразид за участю подвійної кількості гідразин гідрату. Наступна модифікація молекули полягала в поетапному використанні реакцій нуклеофільного приєднання етилізотіоціанату та лужної гетероциклізації. Сформований так 5-(5-метилпіразол)-4-етил-1,2,4-тріазол-3-тіол піддавали процесу алкілування. Для встановлення складу та ідентифікації структури виділених речовин записані ${ }^{1} \mathrm{H}$ ЯMP та інфррачервоні спектри, а також одержані якісні та кількісні показники елементного складу синтезованих структур. Індивідуальний характер наявності речовин і ступінь їхньої чистоти визначені з використанням високоефективної рідинної хроматографрії з двома видами детекції: діодно-матричної та мас-спектрометричної.

Результати. Здійснили синтез алкілпохідних 5-(5-метилпіразол)-4-етил-1,2,4-тріазол-3-тіолу та визначили оптимальні умови процесу одержання цих речовин. Підтверджена будова продуктів хімічного перетворення та зафіксовані результати дослідження фізичних властивостей. Результати докінгових досліджень дали змогу підтвердити перспективність обраного напряму синтетичних перетворень, що врешті дало змогу визначитися з біологічним потенціалом одержаних сполук. Модельні ферменти - анапластичної лімфоми кіназа (код 2XP2), 14-альфа деметилаза ланостеролу (код 3LD6) та циклооксигеназа-1 (код 3N8Y), інформацію щодо яких використано з бази Банку даних білкових структур (PDB).

Висновки. У результаті молекулярного докінгу отримали відомості, що формують уявлення про певний рівень імовірності впливу синтезованих сполук на активність названих фрерментних структур.

Ключові слова: 1,2,4-тріазол, піразол, фрізико-хімічні властивості, молекулярний докінг.

Актуальні питання фрармацевтичної і медичної науки та практики. 2020. Т. 13, № 3(34). С. 318-323

\section{Синтез и свойства некоторых пиразолпроизводных 1,2,4-триазол-3-тиола}

\section{А. С. Гоцуля, С. Н. Кулиш}

Нитрогенсодержащие гетероциклические соединения играют важную роль в современной фармацевтической отрасли. Это связано со значительным биологическим потенциалом указанных соединений. 1,2,4-Триазол и пиразол- известные фармакофоры, которые отвечают за формирование широкого спектра активностей. Конструирование целевой молекулы с использованием 1,2,4-триазола и пиразола интересно с точки зрения доступности исходных реагентов и простоты химических превращений. Совмещение этих гетероциклов в одной молекуле позволяет увеличить возможность участия в разнообразных биологических процессах.

Цель работы - исследование условий получения S-алкилпроизводных 5-(5-метилпиразол)-4-этил-1,2,4-триазол-3-тиола и изучение свойств указанных веществ.

Материалы и методы. Начальный этап работы предусматривал использование диэтилоксалата, ацетона и натрий метилата как исходных веществ для постановки синтеза промежуточного продукта химического преобразования. Полученный метил 2,4-диоксопентаноат на следующем этапе применен в процессе преобразования в 5-метилпиразол-3-карбогидразид при участии двойного количества гидразин гидрата. Последующая модификация молекулы предусматривала использование этилизотиоцианата и щелочную гетероциклизацию. Сформированный таким образом 5-(5-метилпиразол)-4-этил-1,2,4-триазол-3-тиол подвергали процессу алкилирования. Для установления состава и идентификации структуры выделенных веществ записаны ${ }^{1} \mathrm{H}$ ЯМР и инфракрасные спектры, а также получены качественные и количественные показатели элементного состава синтезированных структур. Индивидуальный характер присутствия веществ и степень их чистоты определены с использованием высокоэффективной жидкостной хроматографии с двумя видами детекции: диодно-матричной и масс-спектрометрической.

Результаты. Проведен синтез алкилпроизводных 5-(5-метилпиразол)-4-этил-1,2,4-триазол-3-тиола и определены оптимальные условия процесса получения указанных веществ. Подтверждено строение продуктов химического преобразования, зафиксированы результаты исследования физических свойств. Результаты докинговых исследований позволяют подтвердить перспективность выбранного направления синтетических преобразований, что дало возможность определиться с биологическим потенциалом полученных веществ. Модельные ферменты: анапластической лимфомы киназа (код 2ХР2), 14-альфа деметилаза ланостерола (код 3LD6) и циклооксигеназа-1 (код 3N8Y), информация о которых использована из базы Банка данных белковых структур (PDB).

Выводы. В результате молекулярного докинга получены данные, которые формируют представление про определенный уровень вероятности влияния синтезированных соединений на активность указанных ферментных структур.

Ключевые слова: 5-метилпиразол, 1,2,4-триазол, фризико-химические свойства, молекулярный докинг.

Актуальные вопросы фармацевтической и медицинской науки и практики. 2020. Т. 13, № 3(34). С. 318-323

The search for new biologically active substances is a priority of modern pharmaceutical science [1-6].

Among the great variety of pharmacologically active molecules, special attention is drawn to the products of the processes of combining individual synthons into a more complex molecule [7-9]. For example, pyrazole and 1,2,4-triazole derivatives are well studied and demonstrate a wide range of biological activity. Therefore, a combination of such fragments, which show different mechanisms of interaction with the biological target, within one molecule, is quite interesting and relevant.

\section{Aim}

The aim of the research was to study the conditions for obtaining and studying the properties of S-alkylderivatives of 5-(5-methylpyrazole)-4-ethyl-1,2,4-triazole-3-thiol. 


\section{Materials and methods}

Chemistry. Pyrazole was selected as an intermediate for chemical transformation. This five-membered heterocycle was formed using diethyloxalate, acetone and sodium methanoate. Synthesized 5-methylpyrazole-3-carbohydrazide was used to form a fragment of 1,2,4-triazole. The obtained 5-(5-methylpyrazole)-4-ethyl-1,2,4-triazole-3-thiol was used to carry out S-alkylation reactions using halogenoalkanes.

Properties (physical, chemical) of the produced organic materials have been examined in compliance with articles of the State Pharmacopoeia. The melting temperature has been defined in open capillary tubes ("MP 100 Melting Point Systems" manufactured by Mettler Toledo). Qualitative and quantitative elemental analysis was carried out with the "Vario EL cube" manufactured by Elementar Analysensysteme GmbH. Infrared spectra were obtained with the ALPHA FT-IR Spectrometer manufactured by Bruker. Nuclear magnetic resonance spectra on Hydrogen nuclei 1 (400 MHz) were recorded using the instrument "Varian Mercury $400 \mathrm{MHz}$ " spectrometer. Tetramethylsilane in dimethylsulfoxide- $d_{6}$ solution was used as an internal standard for measuring chemical shifts. Chromatography mass spectra were recorded using the instrument "Agilent 1260 Infinity HPLC" in conjunction with a mass spectrometer "Agilent 6120 " (technique of the ionization - electrospray (ESI)).

Methyl 5-methylpyrazole-3-carboxylate (4). $20 \mathrm{~g} \mathrm{H}_{2} \mathrm{~N}$ $\mathrm{NH}_{2} \times \mathrm{H}_{2} \mathrm{O}(0.4 \mathrm{~mol})$ in $60 \mathrm{ml}$ ethyl alcohol is added with cooling to a solution containing 0.4 mol of methyl 2,4-dioxopentanoate (3) in propan-2-ol. Reaction mixture was heated for 1 hour. Then the alcohol was distilled off under vacuum. The resulting precipitate (99\%) was recrystallized from aqueous ethyl alcohol. Formed solid was dried to generate 4 with a melting point of $82-83{ }^{\circ} \mathrm{C}$ (Fig. 1).

5-Methylpyrazole-3-carbohydrazide (5). A mixture of intermediate $4(58.4 \mathrm{~g})$ and $\mathrm{H}_{2} \mathrm{~N}-\mathrm{NH}_{2} \times \mathrm{H}_{2} \mathrm{O}(25 \mathrm{~g})$ was heated at reflux for 7 hours. After cooling, the precipitate was filtrated and recrystallized from $\mathrm{H}_{2} \mathrm{O}$ (Fig. 1). Yield: $88 \%$; melting point: $153-155^{\circ} \mathrm{C}$; IR $\left(\mathrm{v}, \mathrm{cm}^{-1}\right): 3408-3237\left(\mathrm{NH}, \mathrm{NH}_{2}\right), 1625$ $(\mathrm{C}=\mathrm{O}) ;{ }^{1} \mathrm{H}$ NMR $(\delta, \mathrm{ppm}): 2.24\left(\mathrm{~s}, 3 \mathrm{H}, \mathrm{CH}_{3}\right), 4.32(\mathrm{~s}, 2 \mathrm{H}$, $\left.\mathrm{NH}_{2}\right), 6.37$ (s, 1H, pyrazole, $\left.=\mathrm{CH}-\right), 9.18(\mathrm{~s}, 1 \mathrm{H}, \mathrm{CONH})$, 13.52 (s, 1H, pyrazole, NH). Analytically calculated (\%): C 42.85, H 5.75, N 39.98. Found: C 42.75, H 4.82, N 40.06.

2-(5-Methylpyrazole-3-carbonyl)-N-ethylhydrazine-1-carbothioamide (6). A mixture of intermediate 5 (0.05 mol), 145 $\mathrm{ml}$ of 1,4-dioxane and $65 \mathrm{ml}$ of $\mathrm{H}_{2} \mathrm{O}$ was heated to dissolve the starting compound. Then $\mathrm{C}_{2} \mathrm{H}_{5} \mathrm{NCS}(0.05 \mathrm{~mol})$ was added and the solution was heated to reflux for 1 hour. After cooling, $100 \mathrm{ml}$ of $\mathrm{H}_{2} \mathrm{O}$ was added. The resulting substances was filtered and washed with $\mathrm{H}_{2} \mathrm{O}$ and ethyl alcohol. Then it was crystallized from dimethylformamide (Fig. 1). Yield: $70 \%$; melting point: $263-265^{\circ} \mathrm{C}$; IR $\left(\mathrm{v}, \mathrm{cm}^{-1}\right): 3230(\mathrm{NH})$, $1648(\mathrm{C}=\mathrm{O}) ;{ }^{1} \mathrm{H}$ NMR $(\delta, \mathrm{ppm}): 2.26\left(\mathrm{~s}, 3 \mathrm{H}, \mathrm{CH}_{3}\right), 6.45$ $(\mathrm{s}, 1 \mathrm{H}$, pyrazole, $=\mathrm{CH}-), 8.54(\mathrm{~s}, 1 \mathrm{H}, \mathrm{CONH}), 9.63(\mathrm{~s}, 1 \mathrm{H}$, NHCS), 13.28 (s, pyrazole, NH). Analytically calculated (\%): C 42.28, H 5.77, N 30.81, S 14.11. Found C 42.19, H 5.78, N 30.85, S 14.07.
5-(5-Methylpyrazole-3-yl)-4-ethyl-1,2,4-triazole-3-thiol (7). A mixture of compound $6(0.01 \mathrm{~mol}), \mathrm{NaOH}(0.011 \mathrm{~mol})$ and $60 \mathrm{ml}$ of $\mathrm{H}_{2} \mathrm{O}$ was heated to reflux for 2 hours. After cooling, $2 \mathrm{ml}$ of $\mathrm{CH}_{3} \mathrm{COOH}$ concentrated was added to mixture. The resulting compound 7 was filtered, washed with $\mathrm{H}_{2} \mathrm{O}$ and recrystallized from dimethylformamide (Fig. 1). Yield: $85 \%$; melting point: $310-312{ }^{\circ} \mathrm{C}$; IR $\left(\mathrm{v}, \mathrm{cm}^{-1}\right): 2744(\mathrm{SH}) .{ }^{1} \mathrm{HNMR}(\delta$, ppm): 1.27 (t, $\left.J=6.0 \mathrm{~Hz}, 3 \mathrm{H}, \mathrm{CH}_{2}-\mathrm{CH}_{3}\right), 2.34\left(\mathrm{~s}, 3 \mathrm{H}, \mathrm{CH}_{3}\right), 4.33$ (q, $\left.J=6.2 \mathrm{~Hz}, 2 \mathrm{H}, \mathrm{CH}_{2}-\mathrm{CH}_{3}\right), 6.50(\mathrm{~s}, 1 \mathrm{H}$, pyrazole, $=\mathrm{CH}-)$. Analytically calculated (\%): C 45.92, H 5.30, N 33.47, S 15.32. Found C 46.05, H 5.29, N 33.56, S 15.29.

Alkylation. Compound 7 (0.05 mol) was added to a solution of $0.05 \mathrm{~mol}$ of $\mathrm{NaOH}$ in $35 \mathrm{ml}$ of $\mathrm{H}_{2} \mathrm{O}$, until complete dissolution was reached. Subsequently, a solution of 0.055 mol of the alkylating part in methanol or propan-2-ol was added (Fig. 1). The obtained precipitate was filtered, washed by water and recrystallized with ethyl alcohol to give a white solid (7.1-7.10).

Molecular docking was performed to obtain structural information on the interaction of the synthesized compounds and the corresponding biological structure [10,11]. In order to solve the indicated problem, models of the necessary biological target were used. The source of the indicated biological models was the Protein Database. Kinases of anaplastic lymphoma (ALK) in the complex of crizotinib, 14-alpha demethylase of lanosterol with ketoconazole and cyclooxygenase-1 (COX-1) with diclofenac were used from the base. AUTODOCK was used to carry out the process of attaching the studied ligands to the proteins under consideration. The energy interaction between the ligand and protein, hydrogen bonds and hydrophobic interaction were used to analyze the docking interaction [12-14].

\section{Results}

The synthesis of the compounds 7.1-7.10 has been described in Fig. 1.

3-(5-Methylpyrazol-3-yl)-5-methylthio-4-ethyl-1,2,4triazole (7.1). Yield: $76 \%$; melting point: $164-165{ }^{\circ} \mathrm{C}$; IR $\left(v, \mathrm{~cm}^{-1}\right): 3227(\mathrm{NH}), 1604(\mathrm{C}=\mathrm{N}) ;{ }^{1} \mathrm{H} \mathrm{NMR}(\delta, \mathrm{ppm}): 1.29$ (t, $\left.J=6.2 \mathrm{~Hz}, 3 \mathrm{H}, \mathrm{CH}_{2}-\mathrm{C}_{3}\right), 2.33\left(\mathrm{~s}, 3 \mathrm{H}, \mathrm{CH}_{3}\right), 2.76(\mathrm{~s}$, $\left.3 \mathrm{H}, \mathrm{S}-\mathrm{CH}_{3}\right), 4.35\left(\mathrm{q}, J=6.1 \mathrm{~Hz}, 2 \mathrm{H}, \mathrm{CH}_{2}-\mathrm{CH}_{3}\right), 6.47(\mathrm{~s}, 1 \mathrm{H}$, pyrazole, $=\mathrm{CH}-), 11.73(\mathrm{~s}, 1 \mathrm{H}$, pyrazole, $\mathrm{NH})$. Analytically calculated (\%): C 48.41, H 5.87, N 31.36, S 14.36. Found: C 48.28, H 5.86, N 31.44, S 14.32. ESI-MS: m/z = 223 [M+1], $225[\mathrm{M}+3]$.

3-Ethylthio-5-(5-methylpyrazol-3-yl)-4-ethyl-1,2,4-triazole (7.2). Yield: $77 \%$; melting point: $83-84{ }^{\circ} \mathrm{C}$; IR $\left(v, \mathrm{~cm}^{-1}\right): 3232$ $(\mathrm{NH}), 1599(\mathrm{C}=\mathrm{N}) ;{ }^{1} \mathrm{H}$ NMR $(\delta, \mathrm{ppm}): 1.30(\mathrm{t}, J=6.1 \mathrm{~Hz}, 3 \mathrm{H}$, $\left.\mathrm{CH}_{2}-\mathrm{CH}_{3}\right), 1.44\left(\mathrm{t}, J=5.1 \mathrm{~Hz}, 3 \mathrm{H}, \mathrm{S}-\mathrm{CH}_{2}-\mathrm{CH}_{3}\right), 2.35(\mathrm{~s}, 3 \mathrm{H}$, $\mathrm{CH}_{3}$ ), 3.19-3.21 (m, $\left.J=4.8 \mathrm{~Hz}, 2 \mathrm{H}, \mathrm{S}-\mathrm{CH}_{2}-\mathrm{CH}_{3}\right), 4.35$ (q, $\left.J=6.1 \mathrm{~Hz}, 2 \mathrm{H}, \mathrm{CH}_{2}-\mathrm{CH}_{3}\right), 6.45$ (s, 1H, pyrazole, $\left.=\mathrm{CH}-\right), 11.71$ (s, 1H, pyrazole, NH). Analytically calculated (\%): C 50.61, H 6.37, N 29.51, S 13.51. Found C 50.48, H 6.38, N 29.59, S 13.55. ESI-MS: $\mathrm{m} / \mathrm{z}=237[\mathrm{M}+1], 239[\mathrm{M}+3]$.

3-(5-Methylpyrazol-3-yl)-4-ethyl-5-propylthio-1, 2,4triazole (7.3). Yield: $78 \%$; melting point: $80-82{ }^{\circ} \mathrm{C}$; IR ( $v$, 


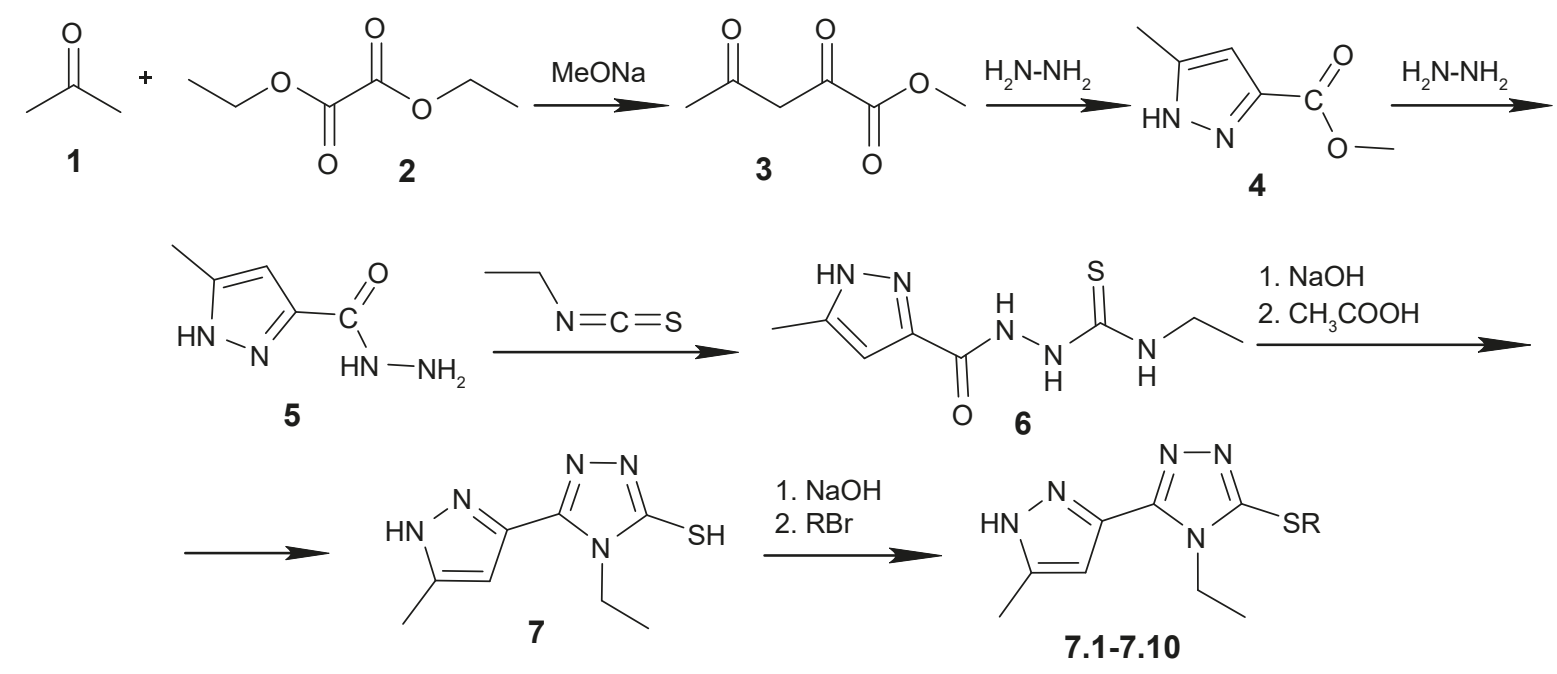

$\mathrm{R}=\mathrm{C}_{\mathrm{n}} \mathrm{H}_{2 \mathrm{n}+1} ; \mathrm{n}=1-10$

Fig. 1. Synthetic pathway for the compounds 7.1-7.10.

$\left.\mathrm{cm}^{-1}\right): 3221(\mathrm{NH}), 1611(\mathrm{C}=\mathrm{N}) ;{ }^{1} \mathrm{H} \operatorname{NMR}(\delta, \mathrm{ppm}): 1.08$ (t, $\left.J=5.4 \mathrm{~Hz}, 3 \mathrm{H}, \mathrm{S}-\left(\mathrm{CH}_{2}\right)_{2}-\underline{C}_{3}\right), 1.32(\mathrm{t}, J=6.1 \mathrm{~Hz}, 3 \mathrm{H}$, $\mathrm{CH}_{2}-\mathrm{CH}_{3}$ ), 1.73-1.76 (m, 2H, S-CH $-\mathrm{CH}_{2}-\mathrm{CH}_{3}$ ), 2.34 (s, $\left.3 \mathrm{H}, \mathrm{CH}_{3}\right), 3.15\left(\mathrm{t}, 2 \mathrm{H}, \mathrm{S}-\mathrm{C}_{2}-\mathrm{CH}_{2}-\mathrm{CH}_{3}\right), 4.35$ (q, J=6.2 Hz, $\left.2 \mathrm{H}, \mathrm{C}_{2}-\mathrm{CH}_{3}\right), 6.47(\mathrm{~s}, 1 \mathrm{H}$, pyrazole, $=\mathrm{CH}-), 11.74(\mathrm{~s}, 1 \mathrm{H}$, pyrazole, NH). Analytically calculated (\%): C 52.56, H 6.82, N 27.86, S 12.76. Found C 52.71, H 5.70, N 27.93, S 10.71. ESI-MS: $\mathrm{m} / \mathrm{z}=251[\mathrm{M}+1], 253[\mathrm{M}+3]$.

3-Butylthio-5-(5-methylpyrazol-3-yl)-4-ethyl-1,2,4triazole (7.4). Yield: $67 \%$; melting point: $74-76^{\circ} \mathrm{C}$; IR ( $v$, $\left.\mathrm{cm}^{-1}\right): 3230(\mathrm{NH}), 1595(\mathrm{C}=\mathrm{N}) ;{ }^{1} \mathrm{H}$ NMR $(\delta, \mathrm{ppm}): 0.84(\mathrm{t}$, $\left.J=5.3 \mathrm{~Hz}, 3 \mathrm{H}, \mathrm{S}-\left(\mathrm{CH}_{2}\right)_{3}-\mathrm{C}_{3}\right), 1.28\left(\mathrm{t}, J=6.1 \mathrm{~Hz}, 3 \mathrm{H}, \mathrm{CH}_{2}-\right.$ $\left.\mathrm{C} \underline{H}_{3}\right), 1.37-1.40\left(\mathrm{~m}, 2 \mathrm{H}, \mathrm{S}-\left(\mathrm{CH}_{2}\right)_{2}-\mathrm{C}_{2}-\mathrm{CH}_{3}\right), 1.68-1.71$ (m, $\left.J=8.16,7.27 \mathrm{~Hz}, 2 \mathrm{H}, \mathrm{S}-\mathrm{CH}_{2}-\mathrm{CH}_{2}-\mathrm{CH}_{2}-\mathrm{CH}_{3}\right), 2.33$ (s, 3H, $\left.\mathrm{CH}_{3}\right), 3.18$ (t, $\left.2 \mathrm{H}, \mathrm{S}-\mathrm{CH}_{2}-\left(\mathrm{CH}_{2}\right)_{2}-\mathrm{CH}_{3}\right), 4.36$ (q, $J=6.0 \mathrm{~Hz}$, $\left.2 \mathrm{H}, \mathrm{CH}_{2}-\mathrm{CH}_{3}\right), 6.46(\mathrm{~s}, 1 \mathrm{H}$, pyrazole, $=\mathrm{CH}-), 11.75(\mathrm{~s}, 1 \mathrm{H}$, pyrazole, NH). Analytically calculated (\%): C 54.31, H 7.22, N 26.39, S 12.08. Found C 54.17, H 7.20, N 26.32, S 12.11. ESI-MS: $\mathrm{m} / \mathrm{z}=265[\mathrm{M}+1], 267[\mathrm{M}+3]$.

3-(5-Methylpyrazol-3-yl)-5-pentylthio-4-ethyl-1,2,4triazole (7.5). Yield: $74 \%$; melting point: $71-73{ }^{\circ} \mathrm{C}$; IR (v, $\left.\mathrm{cm}^{-1}\right): 3221(\mathrm{NH}), 1609(\mathrm{C}=\mathrm{N}) ;{ }^{1} \mathrm{H}$ NMR $(\delta, \mathrm{ppm}): 0.85(\mathrm{t}$, $\left.J=5.3 \mathrm{~Hz}, 3 \mathrm{H}, \mathrm{S}-\left(\mathrm{CH}_{2}\right)_{4}-\mathrm{C}_{3}\right), 1.36-1.41\left(\mathrm{~m}, 7 \mathrm{H}, \mathrm{CH}_{2}-\mathrm{CH}_{3}\right.$, $\left.\mathrm{S}-\left(\mathrm{CH}_{2}\right)_{2}-\left(\mathrm{CH}_{2}\right)_{2}-\mathrm{CH}_{3}\right), 1.66-1.69(\mathrm{~m}, J=7.92,7.25 \mathrm{~Hz}, 2 \mathrm{H}$, $\left.\mathrm{S}-\mathrm{CH}_{2}-\mathrm{CH}_{2}-\left(\mathrm{CH}_{2}\right)_{2}-\mathrm{CH}_{3}\right), 2.33\left(\mathrm{~s}, 3 \mathrm{H}, \mathrm{CH}_{3}\right), 3.15(\mathrm{t}, J=5.1$ $\left.\mathrm{Hz}, 2 \mathrm{H}, \mathrm{S}-\mathrm{CH}_{2}-\left(\mathrm{CH}_{2}\right)_{3}-\mathrm{CH}_{3}\right), 4.37$ (q, $J=6.2 \mathrm{~Hz}, 2 \mathrm{H}, \mathrm{CH}_{2}-$ $\left.\mathrm{CH}_{3}\right), 6.47(\mathrm{~s}, 1 \mathrm{H}$, pyrazole, $=\mathrm{CH}-), 11.74(\mathrm{~s}, 1 \mathrm{H}$, pyrazole, $\mathrm{NH})$. Analytically calculated (\%): C 55.88, H 7.58, N 25.07, S 11.47. Found: C 56.01, H 7.56, N 25.13, S 11.44. ESI-MS: $\mathrm{m} / \mathrm{z}=279[\mathrm{M}+1], 281[\mathrm{M}+3]$.

3-Hexylthio-5-(5-methylpyrazol-3-yl)-4-ethyl-1,2,4triazole (7.6). Yield: $82 \%$; melting point:70-71 ${ }^{\circ} \mathrm{C}$; IR (v, $\left.\mathrm{cm}^{-1}\right): 3235(\mathrm{NH}), 1624(\mathrm{C}=\mathrm{N}) ;{ }^{1} \mathrm{H}$ NMR $(\delta, \mathrm{ppm}): 0.88(\mathrm{t}$, $\left.J=5.4 \mathrm{~Hz}, 3 \mathrm{H}, \mathrm{S}-\left(\mathrm{CH}_{2}\right)_{5}-\mathrm{C}_{3}\right), 1.37-1.42(\mathrm{~m}, J=4.8 \mathrm{~Hz}, 1.7$, $\left.1.3 \mathrm{~Hz}, 9 \mathrm{H}, \mathrm{CH}_{2}-\underline{\mathrm{CH}}_{3}, \mathrm{~S}-\left(\mathrm{CH}_{2}\right)_{2}-\left(\mathrm{C}_{2}\right)_{3}-\mathrm{CH}_{3}\right), 1.72-1.75$ (m, $\left.J=7.8 \mathrm{~Hz}, 2 \mathrm{H}, \mathrm{S}-\mathrm{CH}_{2}-\mathrm{CH}_{2}-\left(\mathrm{CH}_{2}\right)_{3}-\mathrm{CH}_{3}\right), 2.33\left(\mathrm{~s}, 3 \mathrm{H}, \mathrm{CH}_{3}\right)$, 3.18 (t, $\left.J=7.9 \mathrm{~Hz}, 2 \mathrm{H}, \mathrm{S}-\mathrm{C}_{2}-\left(\mathrm{CH}_{2}\right)_{4}-\mathrm{CH}_{3}\right), 4.36$ (q, $J=6.1$ $\left.\mathrm{Hz}, 2 \mathrm{H}, \underline{\mathrm{CH}}_{2}-\mathrm{CH}_{3}\right), 6.47$ (s, $1 \mathrm{H}$, pyrazole, $\left.=\mathrm{CH}-\right), 11.73$ (s, $1 \mathrm{H}$, pyrazole, $\mathrm{NH}$ ). Analytically calculated (\%): C 57.31, H 7.90, N 23.87, S 10.93. Found: C 57.16, H 7.88, N 23.82, S 9.44. ESI-MS: $\mathrm{m} / \mathrm{z}=293\left[\mathrm{M}+\mathrm{H}^{+}\right], 295[\mathrm{M}+3]$.

3-Heptylthio-5-(5-methylpyrazol-3-yl)-4-ethyl-1,2,4triazole (7.7). Yield: $66 \%$; melting point: $69-71{ }^{\circ} \mathrm{C}$; IR $\left(v, \mathrm{~cm}^{-1}\right): 3219(\mathrm{NH}), 1591(\mathrm{C}=\mathrm{N}) ;{ }^{1} \mathrm{H}$ NMR $(\delta, \mathrm{ppm})$ : $0.88\left(\mathrm{t}, 3 \mathrm{H}, \mathrm{S}-\left(\mathrm{CH}_{2}\right)_{6}-\mathrm{C}_{3}\right), 1.33-1.40\left(\mathrm{~m}, 11 \mathrm{H}, \mathrm{CH}_{2}-\mathrm{C}_{3}\right.$, $\left.\mathrm{S}-\left(\mathrm{CH}_{2}\right)_{2}-\left(\mathrm{CH}_{2}\right)_{4}-\mathrm{CH}_{3}\right), 1.70-1.73(\mathrm{~m}, J=8.2 \mathrm{~Hz}, 2 \mathrm{H}$, $\left.\mathrm{S}-\mathrm{CH}_{2}-\left(\mathrm{CH}_{2}\right)_{5}-\mathrm{CH}_{3}\right), 2.31\left(\mathrm{~s}, 3 \mathrm{H}, \mathrm{CH}_{3}\right), 3.18(\mathrm{t}, J=7.9 \mathrm{~Hz}$, $\left.2 \mathrm{H}, \mathrm{S}-\mathrm{C} \underline{H}_{2}-\left(\mathrm{CH}_{2}\right)_{5}-\mathrm{CH}_{3}\right), 4.36\left(\mathrm{q}, J=6.1 \mathrm{~Hz}, 2 \mathrm{H}, \mathrm{CH}_{2}-\mathrm{CH}_{3}\right)$, $6.47(\mathrm{~s}, 1 \mathrm{H}$, pyrazole, $=\mathrm{CH}-), 11.73(\mathrm{~s}, 1 \mathrm{H}$, pyrazole, $\mathrm{NH})$. Analytically calculated (\%): C 58.60, H 8.20, N 22.78, S 10.43. Found C 58.76, H 7.09, N 22.72, S 10.46. ESI-MS: $\mathrm{m} / \mathrm{z}=307\left[\mathrm{M}+\mathrm{H}^{+}\right], 309[\mathrm{M}+3]$.

3-(5-Methylpyrazol-3-yl)-5-octylthio-4-ethyl-1,2,4-triazole (7.8). Yield: $82 \%$; melting point: $66-68{ }^{\circ} \mathrm{C}$; IR $\left(v, \mathrm{~cm}^{-1}\right): 3221$ $(\mathrm{NH}), 1607(\mathrm{C}=\mathrm{N}) ;{ }^{1} \mathrm{H}$ NMR $(\delta, \mathrm{ppm}): 0.89(\mathrm{t}, J=5.4 \mathrm{~Hz}, 3 \mathrm{H}$, $\left.\mathrm{S}-\left(\mathrm{CH}_{2}\right)_{7}-\mathrm{CH}_{3}\right), 1.32-1.38\left(\mathrm{~m}, 13 \mathrm{H}, \mathrm{CH}_{2}-\mathrm{CH}_{3}, \mathrm{~S}-\left(\mathrm{CH}_{2}\right)_{2}-\left(\mathrm{C}_{2}\right)_{5}-\right.$ $\left.\mathrm{CH}_{3}\right), 1.68-1.71\left(\mathrm{~m}, J=8.0 \mathrm{~Hz}, 2 \mathrm{H}, \mathrm{S}-\mathrm{CH}_{2}-\mathrm{CH}_{2}-\left(\mathrm{CH}_{2}\right)_{5}-\mathrm{CH}_{3}\right)$, $2.31\left(\mathrm{~s}, 3 \mathrm{H}, \mathrm{CH}_{3}\right), 3.19$ (t, J=7.8 Hz, $\left.2 \mathrm{H}, \mathrm{S}-\mathrm{CH}_{2}-\left(\mathrm{CH}_{2}\right)_{7}-\mathrm{CH}_{3}\right)$, 4.37 (q, $\left.J=6.2 \mathrm{~Hz}, 2 \mathrm{H}, \mathrm{C}_{2}-\mathrm{CH}_{3}\right), 6.46(\mathrm{~s}, 1 \mathrm{H}$, pyrazole, $=\mathrm{CH}-$ ), 11.73 (s, 1H, pyrazole, NH). Analytically calculated (\%): C 59.78, H 8.47, N 21.78, S 9.97. Found C 59.61, H 8.49, N 21.73, S 9.99. ESI-MS: $\mathrm{m} / \mathrm{z}=321\left[\mathrm{M}+\mathrm{H}^{+}\right], 323[\mathrm{M}+3]$.

3-(5-Methylpyrazol-3-yl)-5-nonylthio-4-ethyl-1,2,4-triazole (7.9). Yield: $69 \%$; melting point: $62-63{ }^{\circ} \mathrm{C}$; IR $\left(v, \mathrm{~cm}^{-1}\right): 3227$ $(\mathrm{NH}), 1584(\mathrm{C}=\mathrm{N}) ;{ }^{1} \mathrm{H}$ NMR $(\delta, \mathrm{ppm}): 0.89(\mathrm{t}, J=5.4 \mathrm{~Hz}, 3 \mathrm{H}$, $\left.\mathrm{S}-\left(\mathrm{CH}_{2}\right)_{8}-\mathrm{CH}_{3}\right), 1.32-1.38\left(\mathrm{~m}, 15 \mathrm{H}, \mathrm{CH}_{2}-\mathrm{CH}_{3}, \mathrm{~S}-\left(\mathrm{CH}_{2}\right)_{2}-\left(\mathrm{CH}_{2}\right)_{6}-\right.$ $\left.\mathrm{CH}_{3}\right), 1.68-1.71\left(\mathrm{~m}, J=8.2 \mathrm{~Hz}, 2 \mathrm{H}, \mathrm{S}-\mathrm{CH}_{2}-\mathrm{CH}_{2}-\left(\mathrm{CH}_{2}\right)_{6}-\mathrm{CH}_{3}\right)$, $2.32\left(\mathrm{~s}, 3 \mathrm{H}, \mathrm{CH}_{3}\right), 3.17\left(\mathrm{t}, J=7.9 \mathrm{~Hz}, 2 \mathrm{H}, \mathrm{S}-\mathrm{C}_{2}-\left(\mathrm{CH}_{2}\right)_{7}-\mathrm{CH}_{3}\right)$, 
Table 1. Molecular docking calculation with ALK

\begin{tabular}{|l|l|l|l|l|l|}
\hline № & $E_{\text {min }}, \mathrm{kcal} / \mathrm{mol}$ & № & $E_{\min }, \mathrm{kcal} / \mathrm{mol}$ & № & $E_{\text {min }}, \mathrm{kcal} / \mathrm{mol}$ \\
\hline 6 & -5.4 & 7.4 & -7.6 & 7.8 & -7.8 \\
\hline 7.1 & -6.1 & 7.5 & -7.7 & 7.9 & -7.7 \\
\hline 7.2 & -6.1 & 7.6 & -7.4 & 7.10 & -7.5 \\
\hline 7.3 & -7.5 & 7.7 & -7.7 & Crizotinib & -9.4 \\
\hline
\end{tabular}

$E_{\text {min }}:$ the minimum interaction energy, $\mathrm{kcal} / \mathrm{mol}$.

Table 2. Molecular docking calculation with COX-1

\begin{tabular}{|l|l|l|l|l|l|}
\hline № & $E_{\min }, \mathrm{kcal} / \mathrm{mol}$ & № & $E_{\min }, \mathrm{kcal} / \mathrm{mol}$ & № & 7.8 \\
\hline 6 & -8.8 & 7.4 & -6.2 & 7.9 & -6.9 \\
\hline 7.1 & -5.3 & 7.5 & -6.2 & 7.9 & -7.3 \\
\hline 7.2 & -5.4 & 7.6 & -6.3 & -10 & -7.4 \\
\hline 7.3 & -5.6 & 7.7 & -6.5 & Diclofenac & $-19,9$ \\
\hline
\end{tabular}

$E_{\text {min }}:$ the minimum interaction energy, $\mathrm{kcal} / \mathrm{mol}$.

Table 3. Molecular docking calculation with lanosterol 14a-demethylase

\begin{tabular}{|c|c|c|c|c|c|}
\hline N & $E_{\min }, \mathrm{kcal} / \mathrm{mol}$ & $\mathbf{N}$ & $E_{\min }, \mathrm{kcal} / \mathrm{mol}$ & $\mathbf{N}$ & $E_{\min }, \mathrm{kcal} / \mathrm{mol}$ \\
\hline 6 & -6.0 & 7.4 & -8.5 & 7.8 & -9.5 \\
\hline 7.1 & -6.1 & 7.5 & -8.5 & 7.9 & -9.3 \\
\hline 7.2 & -6.4 & 7.6 & -8.4 & 7.10 & -9.2 \\
\hline 7.3 & -8.5 & 7.7 & -8.5 & Ketoconazole & -10.1 \\
\hline
\end{tabular}

$E_{\min }:$ the minimum interaction energy, $\mathrm{kcal} / \mathrm{mol}$.

$4.37\left(\mathrm{q}, J=6.0 \mathrm{~Hz}, 2 \mathrm{H}, \underline{\mathrm{C}}_{2}-\mathrm{CH}_{3}\right), 6.46(\mathrm{~s}, 1 \mathrm{H}$, pyrazole, $=\mathrm{CH}-$ ), 11.72 (s, 1H, pyrazole, NH). Analytically calculated (\%): C 60.86, H 8.71, N20.87, S 9.56. Found C 60.71, H 8.69, N 20.82, S 9.58. ESI-MS: $\mathrm{m} / \mathrm{z}=335\left[\mathrm{M}+\mathrm{H}^{+}\right], 337[\mathrm{M}+3]$.

3-Decylthio-5-(5-methylpyrazol-3-yl)-4-ethyl-1,2,4-triazole (7.10). Yield: $86 \%$; melting point: $60-61^{\circ} \mathrm{C}$; IR $\left(v, \mathrm{~cm}^{-1}\right): 3233$ $(\mathrm{NH}), 1610(\mathrm{C}=\mathrm{N}) ;{ }^{1} \mathrm{H} \mathrm{NMR}(\delta, \mathrm{ppm}): 0.91(\mathrm{t}, J=5.4 \mathrm{~Hz}, 3 \mathrm{H}$, $\left.\mathrm{S}-\left(\mathrm{CH}_{2}\right)_{9}-\mathrm{CH}_{3}\right), 1.26-1.35\left(\mathrm{~m}, 17 \mathrm{H}, \mathrm{CH}_{2}-\mathrm{CH}_{3}, \mathrm{~S}-\left(\mathrm{CH}_{2}\right)_{2}-\left(\mathrm{C}_{2}\right)_{7}-\right.$ $\left.\mathrm{CH}_{3}\right), 1.66-1.70\left(\mathrm{~m}, J=8.1 \mathrm{~Hz}, 2 \mathrm{H}, \mathrm{S}-\mathrm{CH}_{2}-\mathrm{CH}_{2}-\left(\mathrm{CH}_{2}\right)_{7}-\mathrm{CH}_{3}\right)$, $2.33\left(\mathrm{~s}, 3 \mathrm{H}, \mathrm{CH}_{3}\right), 3.09-3.13\left(\mathrm{t}, J=7.6 \mathrm{~Hz}, 2 \mathrm{H}, \mathrm{S}-\mathrm{C}_{2}-\left(\mathrm{CH}_{2}\right)_{8}-\right.$ $\mathrm{CH}_{3}$ ), 4.38 (q, $\left.J=6.1 \mathrm{~Hz}, 2 \mathrm{H}, \underline{\mathrm{CH}}_{2}-\mathrm{CH}_{3}\right), 6.49$ (s, $1 \mathrm{H}$, pyrazole, $=\mathrm{CH}-), 11.75(\mathrm{~s}, 1 \mathrm{H}$, pyrazole, $\mathrm{NH})$. Analytically calculated $(\%)$ : C 61.85, H 8.94, N 20.04, S 9.17. Found C 61.68, H 8.92, N 20.09, S 9.19. ESI-MS: $\mathrm{m} / \mathrm{z}=349\left[\mathrm{M}+\mathrm{H}^{+}\right], 351[\mathrm{M}+3]$.

Hydrogen atoms of the thioalkyl fragment generate signals in the strong part of the magnetic field [9]. For example, the Hydrogen signal of the thiomethyl group appears as a singlet at $2.76 \mathrm{ppm}$ (7.1). The lengthening of the alkyl chain first leads to a shift in the signal of the methyl group to a stronger part of the magnetic field (up to $\mathrm{S}_{-} \mathrm{C}_{4} \mathrm{H}_{9}$ ). Further elongation of the alkyl fragment $\left(\mathrm{S}-\mathrm{C}_{5} \ldots \mathrm{C}_{10}\right)$ does not cause significant shifts in the Hydrogen signals of the methyl group. The methylene proton signals are observed in the region of strong fields in the form of a triplet at 3.09-3.19 ppm or multiplets at $1.26-1.42 \mathrm{ppm}$ and at $1.66-1.76 \mathrm{ppm}$.

In the IR-spectrum of compounds 7.1-7.10 deformation vibrations of alkyl groups in ranges from $655 \mathrm{~cm}^{-1}$ to $1270 \mathrm{~cm}^{-1}$ and $\mathrm{H}-\mathrm{C}-\mathrm{H}$ fragment in a narrow area of frequency 1480-1365 $\mathrm{cm}^{-1}$ are observed. For example, for $\mathrm{CH}_{3}$-group $\delta$-vibrations occupied an area of 1373-1378 $\mathrm{cm}^{-1}$. Valence vibrations of bonds of C-H alkyl groups form bands in the area of $3075-2845 \mathrm{~cm}^{-1}$.

In the mass spectrum, there is a peak of the molecular ion and peaks of fragment ions, which confirm this structure.

The methodology for rational drug development involves the use of molecular docking. Docking experiments of synthesized compounds $(7,7.1-7.8)$ with the 2XP2 (anaplastic lymphoma receptor tyrosine kinase) receptor revealed that compound 7.8 is the most active with a calculated binding energy of $8.1 \mathrm{kcal} / \mathrm{mol}$ (Table 1) [6,8-10].

It is known about the manifestation of anti-inflammatory activity, which is associated with the combination of triazole and pyrazole fragments within one molecule. In addition, it has been established which aminoacid residues of cyclooxygenases are directly responsible for the formation of hydrogen intermolecular bonds with substances that demonstrate biological activity. Thus, this area of research involving S-alkylderivatives of 5-(5-methylpyrazol-3-yl)-4-ethyl-1,2,4triazole-3-thiol is quite interesting. Cyclooxygenase-1 was chosen as the model enzyme (Table 2).

The effect of 1,2,4-triazole derivatives on the activity of lanosterol 14-alpha demethylase is a proven fact. It is established that 1 and 2 Nitrogen atoms of 1,2,4-triazole fragment are responsible for the formation of $\pi$ - $\pi$-interaction with the active center of the specified enzyme. Therefore, a docking 
study of the effect of synthesized compounds on this enzyme was considered relevant and was carried out (Table 3).

\section{Discussion}

According to the docking results, the synthesized compounds show different levels of binding to the aminoacid residues of anaplastic lymphoma kinase, cyclooxygenase-1 and lanosterol-14 $\alpha$-demethylase.

The transition from thiol to its alkyl derivatives in a number of synthesized compounds leads to an increase in the level of binding to the active centers of anaplastic lymphoma kinase and lanosterol-14 $\alpha$-demethylase.

Docking to cyclooxygenase-1 revealed a decrease in the interaction energy with the specified enzyme of the synthesized alkyl derivatives in comparison with the original thiol. The most relevant for further studies was 3-(5-methylpyrazol-3yl)-5-octylthio-4-ethyl-1,2,4-triazole.

\section{Conclusions}

1. The optimal conditions for obtaining S-alkylderivatives of 5-(5-methylpyrazole)-4-ethyl-1,2,4-triazole-3-thiol were determined. It was found that the highest yield of products of the alkylation reaction of 5-(5-methylpyrazole-3-yl)-4ethyl-1,2,4-triazole-3-thiol was observed when methanol and propan-2-ol were used as solvents.

2. As a result of the molecular docking of the synthesized compounds, promising structures for further studies of anti-inflammatory and antifungal activity were identified. The most promising object for further research is 3-(5-methylpyrazol3-yl)-5-octylthio-4-ethyl-1,2,4-triazole.

Prospects for further research. According to the research results, it is planned to expand classes of compounds to identify promising biologically active compounds among them.

\section{Funding}

The research is carried out within the research topic of Zaporizhzhia State Medical University "Synthesis, physicochemical and biological properties of 3,4-disubstituted 3(5)-thio-1,2,4-triazole with antioxidant, antihypoxic, antimicrobial, cardio and hepatoprotective action", State registration number 0118 U007143 (2018-2022).

Conflicts of interest: authors have no conflict of interest to declare. Конфлікт інтересів: відсутній.

\section{Information about authors:}

Hotsulia A. S., PhD, Associate Professor of the Department of Natural Sciences for Foreign Students and Toxicological Chemistry, Zaporizhzhia State Medical University, Ukraine.

ORCID ID: 0000-0001-9696-221X

Kulish S. M., PhD, Associate Professor of the Department of Natural Sciences for Foreign Students and Toxicological Chemistry, Zaporizhzhia State Medical University, Ukraine.

\section{Відомості про авторів:}

Гоцуля А. С., канд. фрарм. наук, доцент каф. природничих дисциплін для іноземних студентів та токсикологічної хімії, Запорізький державний медичний університет, Україна.
Куліш С. М., канд. фрарм. наук, доцент каф. природничих дисциплін для іноземних студентів та токсикологічної хімії, Запорізький державний медичний університет, Україна.

\section{Сведения об авторах:}

Гоцуля А. С., канд. фрарм. наук, доцент каф. естественных дисциплин для иностранных студентов и токсикологической химии, Запорожский государственный медицинский университет, Украина.

Кулиш С. Н., канд. фрарм. наук, доцент каф. естественных дисциплин для иностранных студентов и токсикологической химии, Запорожский государственный медицинский университет, Украина.

\section{References}

[1] Boraei, A. T. A., El Ashry, E. S. H., \& Duerkop, A. (2016). Regioselectivity of the alkylation of S-substituted 1,2,4-triazoles with dihaloalkanes. Chemistry Central Journal, 10, 22. https://doi.org/10.1186/ s13065-016-0165-0

[2] El-Shaieb, K. M., Mohamed, A. H., \& Abdel-latif, F. F. (2019). Investigation of the reactivity of 4-amino-5-hydrazineyl-4H-1,2,4-triazole-3thiol towards some selected carbonyl compounds: synthesis of novel triazolotriazine-, triazolotetrazine-, and triazolopthalazine derivatives. Zeitschrift Für Naturforschung B, 74(11-12), 847-855. https://doi. org/10.1515/znb-2019-0140

[3] Can, N. Ö., Acar Çevik, U., Sağlık, B. N., Levent, S., Korkut, B., Özkay, Y., \& Koparal, A. S. (2017). Synthesis, Molecular Docking Studies, and Antifungal Activity Evaluation of New Benzimidazole-Triazoles as Potential Lanosterol 14a-Demethylase Inhibitors. Journal of Chemistry, 2017. https://doi.org/10.1155/2017/9387102

[4] Backer, M. M. E., McSweeney, S., Lindley, P. F., \& Hough, E. (2004). Ligand-binding and metal-exchange crystallographic studies on shrimp alkaline phosphatase. Acta Crystallographica Section D-Structural Biology, 60, 1555-1561. https://doi.org/10.1107/s0907444904015628

[5] Kaur, R., Dwivedi, A. R., Kumar, B., \& Kumar, V. Recent (2016). Developments on 1,2,4-triazole nucleus in anticancer compounds. Anti-Cancer Agents in Medicinal Chemistry, 16(4), 465-489. https:// doi.org/10.2174/1871520615666150819121106

[6] Kerru, N., Gummidi, L., Maddila, S., Gangu, K. K., \& Jonnalagadda, S. B. (2020). A review on recent advances in Nitrogen containing molecules and their biological applications. Molecules, 25(8), 1909. https://doi.org/10.3390/molecules25081909

[7] Gotsulya, A. S., Panasenko, O. I., Knysh, Ye. G., \& Knyazevich, P. S (2015). Synthesis and physical-chemical research of 7-((3-thio-4-R$4 \mathrm{H}-1,2,4$-triazole-5-yl)methyl)theophylline carbonyl derivatives. Zaporozhye medical journal, (3), 103-107. https://doi.org/10.14739/23101210.2015.3.44510

[8] Karpun, Ye. O., Karpenko, Yu. V., Parchenko, M. V., \& Bihdan, O. A (2020). Molekuliarnyi dokinh i biodostupnist S-alkilpokhidnykh 5-(3-fluorofenil)-, 5-(5-bromofuran-2-il)- ta 5-(((3-(pirydyn-4-il)-1H-1,2,4triazol-5-il)tio)metyl)-4-metyl-4H-1,2,4-triazoliv in silico metodamy [Molecular docking and bioavailability of S-alkyl derivatives 5-(3-fluorophenyl)-, 5-(5-bromofuran-2-yl)- and -(((3-(pyridin-4-yl)-1H-1,2,4-triazole-5-yl))thio)methyl)-4-methyl-4H-1,2,4-triazole in silico methods]. Current issues in pharmacy and medicine: science and practice, 13(1), 38-45. http://dx.doi.org/10.14739/2409-2932.2020.1.198122

[9] El-Sherief, H., Youssif, B., Abbas Bukhari, S. N., Abdelazeem, A. H., Abdel-Aziz, M., \& Abdel-Rahman, H. M. (2018). Synthesis, anticancer activity and molecular modeling studies of 1,2,4-triazole derivatives as EGFR inhibitors. European journal of medicinal chemistry, 156, 774-789. https://doi.org/10.1016/j.ejmech.2018.07.024

[10] Filimonov, D. A., Druzhilovskiy, D. S., Lagunin, A. A., Gloriozova, T. A. Rudik, A. V., Dmitriev, A. V., Pogodin P. V., \& Poroikov, V. V. (2018). Komp'yuternoe prognozirovanie spektrov biologicheskoi aktivnosti khimicheskikh soedinenii: vozmozhnosti i ogranicheniya [Computer-aided prediction of biological activity spectra for chemical compounds: opportunities and limitations]. Biomedical Chemistry: Research and Methods, 1(1), e00004. [in Russian]. https://doi.org/10.18097/bmcrm00004

[11] Biovia. (2019). Discovery Studio Visualizer, v 19.1.0.18287 [Software]. http://www.3dsbiovia.com/

[12] ChemAxon. (2015). MarvinSketch, Version 6.3.0. [Software]. http:// www.chemaxon.com

[13] Worldwide Protein Data Bank. (n.d.). Protein Data Bank (PDB) [Database]. http://www.pdb.org 